\title{
INTERACTION BETWEEN DISGUST PRONENESS AND PERCEPTION OF TREATMENT EFFICACY PREDICTS RESPONSE TO A DISGUST PLACEBO
}

\author{
Saša Zorjan ${ }^{1,2}$, Andreas Gremsl ${ }^{1}$ \& Anne Schienle ${ }^{1}$ \\ ${ }^{I}$ Department of Clinical Psychology, University of Graz, Graz, Austria \\ ${ }^{2}$ Department of Psychology, University of Maribor, Maribor, Austria
}

received: 7.11.2017;

revised: 10.10.2018;

accepted: 14.11 .2018

\section{SUMMARY}

Placebo responsiveness is highly variable across individuals and has been shown to be difficult to predict solely based on personality traits. The present study examined the interaction between a specific trait and state variable (disgust proneness and perceived effectiveness of a disgust placebo) on placebo responsiveness. We presented 145 participants with an inert pill, which was introduced as an effective anti-nausea drug. Disgust responses were elicited by a validated picture set, which was viewed once with and once without the disgust placebo. The results showed that the placebo was effective in reducing experienced disgust. When predicting placebo responsiveness, the results showed a significant interaction between perceived treatment effectiveness and disgust proneness. Specifically, effectiveness ratings were a significant predictor of placebo response only for individuals high in disgust proneness. The results suggest that the joint consideration of specific state and trait factors can be used to optimize placebo responsiveness.

Key words: disgust - placebo - treatment efficacy - treatment response

\section{INTRODUCTION}

The placebo effect refers to the benefit experienced by patients that arises solely from the knowledge that a treatment (which is actually neutral, inert or inactive) has been given (Moerman \& Jonas 2002). There is great variability in placebo responsiveness. The variability is present both in terms of the magnitude of the placebo effect in different study designs (Weimer et al. 2015) and also in terms of interpersonal differences (Colagiuri et al. 2015). Some authors have postulated that the latter could partially be attributed to personality traits (Corsi \& Colloca 2017, Jakšić et al. 2013).

The idea that personality traits moderate the placebo response has been studied in the past across several placebo domains. Among others, dispositional optimism (Geers et al. 2010), fear of pain (Lyby et al. 2010) and reward sensitivity (Schweinhardt et al. 2009) have all been shown to predict the placebo response. However, for some of these traits, replications were not successful (e.g., van Laarhoven et al. 2011). Moreover, many of the earlier studies did not include a control no-placebo condition, making it difficult to attribute the effects solely to the placebo manipulation (Horing et al. 2014).

The heterogeneous findings prompted researchers to integrate more cognitive trait factors, such as self-efficacy, locus of control and also state factors (e.g., expectations about treatment effectiveness) in the analysis (Corsi \& Colloca 2017, Horing et al. 2014). For example, studies have shown that personality factors, such as dispositional optimism and pessimism, interact with expectations about placebo and nocebo effects (Geers et al. 2005). More specifically, pessimists showed stronger responses to nocebo suggestions than optimists. Corsi and Calloca (2017) tested whether the interplay between trait and state factors could be explained by mediation and tested whether expectations mediate the relationship between psychological traits and placebo responsiveness but did not find support for that hypothesis. The exact relationship between trait and state factors in predicting placebo responsiveness is thus still not clear and needs further research.

As mentioned previously, another big source of variability in findings also stems from the differences between the various designs of the placebo experiments, which include studies on pain analgesia, motion sickness and sound aversion. It has been shown in the past that placebo responses vary greatly depending on the disease or symptom that is being treated as well as on the selected treatment method and setting (Weimer et al. 2015). Thus, different experimental designs induce different placebo responses, which are possibly moderated by different personality traits. It is therefore possible, that study-specific personality traits have to be investigated together with specific state factors (e.g., expectations) to uncover more reliable predictors for the placebo response.

The present analysis focused on one specific placebo design. The participants were all treated with a disgust placebo, which had been introduced as an effective antinausea drug. Disgust responses were elicited by means of a validated picture set, which was once viewed with and once without the placebo. We then examined whether the interaction between study-relevant personality traits (i.e., disgust proneness) and perceived treatment efficacy of a disgust placebo could explain individual differences in placebo responsiveness. 


\section{SUBJECTS AND METHODS}

A total of 145 healthy women $\left(\mathrm{M}_{\mathrm{age}}=23.14, \mathrm{SD}=4.33\right)$ from four studies using a disgust placebo were included in the analysis (Schienle et al. 2016a, 2014, Schienle et al. 2016b, unpublished data). All participants viewed disgusting and neutral pictures. The disgust scenes showed repulsive animals (e.g., maggots, snails), poor hygiene (e.g., dirty toilet, garbage) and unusual/spoiled food (e.g., a man eats a grasshopper). The neutral pictures depicted nature scenes and household items. During the no-placebo condition the disgust images were able to induce the target emotion (i.e., disgust) to a significantly higher degree $(\mathrm{M}=6.77, \mathrm{SD}=1.74)$ than fear $(\mathrm{M}=2.60, \mathrm{SD}=1.74$, $\mathrm{t}(144)=26.78, \mathrm{p}<0.001, \mathrm{~d}=2.22)$, and can therefore be considered specific disgust elicitors. The participants were asked to rate the intensity of elicited emotions for the pictures on 9-point Likert scales $(1=$ little; $9=$ very intense).

All participants underwent two sessions during which they passively viewed the pictures. In the placebo condition, the participants received a placebo pill prior to the presentation of the pictures. They were told that this is an herbal medicine, which has previously been shown to be effective in the treatment of disgustrelated symptoms. In the no-placebo condition, the participants viewed the same pictures without receiving the placebo. The sequence of the pictures within one session was randomized. The order of the two sessions (Placebo, No-placebo) was counterbalanced across participants. The studies have been approved by the ethics committee of the University of Graz, Austria.

\section{Measures}

The QADP (The Questionnaire for the Assessment of Disgust Proneness; Schienle, Walter, Stark, \& Vaitl, 2002), describes 37 disgust-relevant situations ("You touch the toilet seat with part of your body in a public restroom") which have to be rated on 5-point scales from "not disgusting" (0) to "very disgusting" (4). The internal consistency (Cronbach's alpha) in the current sample was 0.88 .

The SADS (a scale for assessing disgust sensitivity; Schienle et. al. 2010) is a self-report measure with 7 items (e.g., experiencing disgust is stressful for me). Disgust sensitivity refers to difficulties to regulate one's own disgust feelings. The internal consistency in the current sample was 0.83 .

Perceived effectiveness of placebo treatment. After the placebo condition, participants were asked to rate the perceived effectiveness of the placebo. This was assessed with a single question: "How effective was the pill you were given". The participants rated this question on a 9 -point Likert scale $(1=$ not at all, $9=$ very).

\section{Statistical analysis}

In order to examine the predictive role of age, disgust proneness (DP), disgust sensitivity (DS) and perceived disgust-placebo effectiveness on the placebo response, a multiple regression analysis, with the enter method was conducted. Placebo response was defined as the difference in disgust ratings (i.e., disgust no placebo - disgust placebo). A higher score represents a greater reduction in disgust ratings in the placebo condition, relative to the no placebo condition. We started with a full factorial model (i.e., all main effects and all possible interactions). To avoid mutlicolinearity, all predictors were mean centered before they were entered into the model. We then removed the highest-order nonsignificant terms by the means of backwards elimination. Due to the statistically significant interaction between DP and effectiveness ratings we analysed the effects of placebo effectiveness ratings on the placebo response separately for different levels of DP (i.e., $1 \mathrm{SD}$ below mean and 1 SD above mean), by examining simple slopes. We assessed the final model for multicolinearity and examined the standardized residuals, Cook's distances and the variance inflation factor, which all indicated a good fit of our model to the data. The analyses were conducted using SPSS version 24 .

\section{RESULTS}

\section{Manipulation check}

In order to check whether the disgust placebo was effective in reducing disgust ratings, we conducted a 2-way ANOVA with factors Treatment (No-placebo, Placebo) and Picture category (Disgust, Neutral). Disgust ratings were entered as the dependent variable. The results showed that there was a significant interaction between Treatment and Category $(\mathrm{F}(1,144)=199.79$, $\mathrm{p}<0.001)$. Follow-up t-tests showed that for neutral pictures the disgust ratings lowered slightly from the noplacebo condition $(\mathrm{M}=1.19, \mathrm{SD}=0.60)$ to the placebo condition $(\mathrm{M}=1.06, \mathrm{SD}=0.26), \mathrm{t}(144)=2.84, \mathrm{p}=0.005$, $\mathrm{d}=0.25$ ). For disgust pictures the decrease in disgust ratings from the no-placebo condition $(\mathrm{M}=6.77$, $\mathrm{SD}=1.74)$ to the placebo condition $(\mathrm{M}=3.78, \mathrm{SD}=2.05)$ was much larger $(\mathrm{t}(144)=15.70, \mathrm{p}<0.001, \mathrm{~d}=1.31)$.

\section{Predicting the placebo response}

The average decrease in disgust ratings from noplacebo to placebo was $2.99(\mathrm{SD}=2.30)$. The participants rated the effectiveness of the administered disgust placebo with $\mathrm{M}=5.96(\mathrm{SD}=2.05)$. The mean DP score was $2.53(\mathrm{SD}=0.51)$ and the mean $\mathrm{DS}$ score was 1.08 $(\mathrm{SD}=0.73)$. The placebo response correlated positively with effectiveness ratings $(\mathrm{r}=0.47, \mathrm{p}<0.001)$ and $\mathrm{DP}$ $(\mathrm{r}=0.28, \mathrm{p}<0.01)$. There was a positive correlation between DP and effectiveness ratings $(r=0.29, \mathrm{p}<0.01)$. Correlations between placebo response and age $(\mathrm{r}=0.10)$ and DS ( $r=0.01)$ were not statistically significant $(\mathrm{p}>0.05)$. 
Table 1. Multiple regression results summary for predicting the placebo response

\begin{tabular}{lccc}
\hline Predictors & B & $95 \%$ CI for B & $\beta$ \\
\hline DP & 0.93 & $0.27-1.58$ & $0.21^{*}$ \\
Effectiveness rating & 0.47 & $0.30-0.63$ & $0.42^{* *}$ \\
DP x Effectiveness rating & 0.55 & $0.26-0.83$ & $0.27^{* *}$ \\
\hline
\end{tabular}

Note. $\mathrm{DP}=$ disgust proneness; $\mathrm{CI}=$ confidence interval; $\quad$ Total $\mathrm{R}^{2}=0.32 ; * \mathrm{p}<0.01, * * \mathrm{p}<0.001$

We examined the predictive role of age, DP, DS and effectiveness ratings on the placebo response by multiple regression. Age $(\beta=0.07, \mathrm{t}(139)=0.99, \mathrm{p}=0.32)$ and DS $(\beta=-0.05, \mathrm{t}(139)=-0.70, \mathrm{p}=0.48)$ were not predictive for the placebo response. All interactions containing these two variables were also not predictive (all p's $>0.29$ ). The final model (Table 1) showed that the interaction between DP and the perceived disgust-placebo effectiveness was a statistically significantly predictor of the placebo response $(\beta=0.27, \mathrm{t}(141)=3.75, \mathrm{p}<0.001)$.

We therefore examined simple slopes (i.e., the effects of placebo effectiveness ratings at different levels of DP). More specifically, we examined the effects of perceived effectiveness at 1 SD above the DP mean and at $1 \mathrm{SD}$ below the DP mean. The simple slope analysis showed that the perceived effectiveness was predictive of the placebo response (i.e., disgust reduction in placebo relative to no placebo condition) at $1 \mathrm{SD}$ above the DP mean (i.e., high levels of disgust proneness) $(\beta=0.67$, $\mathrm{t}(141)=6.79, \mathrm{p}<0.001$ ). At 1 SD below DP mean (i.e., low levels of disgust proneness), the perceived placebo effectiveness was no longer a statistically significant predictor $(\beta=0.17, \mathrm{t}(141)=1.66, \mathrm{p}=0.10)$.

\section{DISCUSSION}

The present study examined the combined role of trait disgust and perceived disgust-placebo effectiveness on the placebo response. We focused on specific personality characteristics (disgust proneness/ sensitivity in a study of a disgust placebo) and examined their interaction with a specific state variable (i.e., perceived effectiveness of the treatment). The results showed that the interaction between the effectiveness ratings and DP was able to predict the placebo response. More specifically, we found that effectiveness ratings were a positive predictor of placebo responsiveness (i.e., reduction of disgust ratings in the placebo condition relative to the no-placebo condition), but only for people high in DP.

Overall, these results are in line with studies that found an interaction between state and trait factors when predicting the placebo response (Geers et al. 2007). Reward expectations (e.g., expectations of improvement) have been postulated as an important mechanism in the placebo response (Enck, Benedetti, \& Schedlowski, 2008). For example, a study by Scott and colleagues (2007) found that the anticipation of a placebo treatment was associated with the activation of dopamingeric neurotransmission and D2/D3 receptors. The change was proportional to the anticipated effects of the placebo. Moreover, the study found that individuals with a greater activation of dopaminergic activation in the nucleus accumbens showed larger placebo responses. Extending this to our study, a possible explanation of the interaction found would be that disgust-prone participants expected the placebo to be more effective due to the fact that the placebo was a disgust placebo, with high relevance for them. This was also evident in the effectiveness ratings, which were correlated positively with DP. Furthemore, DP correlated positively with reported disgust during the no-placebo condition $(\mathrm{r}=0.48, \mathrm{p}<0.001)$, indicating a tendency for disgustprone individuals to react more intensely to the disgusting images. This is also in line with previous findings (Schienle et al. 2002).

There are some limitations that need to be considered when interpreting the results. First, the included studies used a laboratory-based design on one specific domain 'disgust', therefore the results cannot be generalized to other domains. The participants were predominantly college students and all female (due to the greater disgust proneness of women), which also limits generalizability. It will be critical for future research to replicate our findings and also include clinical samples and to establish greater ecological validity (e.g., real exposure to disgust elicitors).

\section{CONCLUSIONS}

Overall, this study found evidence for a specific interplay of state/trait-related disgust variables in predicting the disgust placebo response. We showed that the perception of treatment efficacy played an important role in placebo responsiveness only in disgust-prone individuals. Extending this finding to other placebo domains might help to enhance placebo effects. More specifically, the joint consideration of specific state and trait factors could be used to optimize placebo responsiveness.

\section{Acknowledgements:}

This work was supported by the Austrian Science Fund P 27388-B31.

Conflict of interest: None to declare.

\section{Contribution of individual authors:}

Saša Zorjan contributed to data preparation, data analysis and manuscript preparation;

Andreas Gremsl contributed to data preparation and manuscript preparation;

Anne Schienle contributed to study conceptualization, data collection, data preparation, data analysis and manuscript preparation. 


\section{References}

1. Colagiuri B, Schenk LA, Kessler MD, Dorsey SG, Colloca L: The placebo effect: From concepts to genes. Neuroscience 2015; 307:171-190. doi:10.1016/j.neuroscience.2015.08.017

2. Corsi N, Colloca L: Placebo and Nocebo Effects: The Advantage of Measuring Expectations and Psychological Factors. Front Psychol 2017; 8:308. doi:10.3389/fpsyg.2017.00308.

3. Enck P, Benedetti F, Schedlowski M: New Insights into the Placebo and Nocebo Responses. Neuron 2008; 59:195206. doi:10.1016/j.neuron.2008.06.030.

4. Geers AL, Kosbab K, Helfer SG, Weiland PE, Wellman JA: Further evidence for individual differences in placebo responding: An interactionist perspective. J Psychosom Res 2007; 62:563-570. doi:10.1016/j.jpsychores.2006.12.005

5. Geers AL, Wellman JA, Fowler SL, Helfer SG, France CR: Dispositional Optimism Predicts Placebo Analgesia. J Pain 2010; 11:1165-1171. doi:10.1016/j.jpain.2010.02.014

6. Horing B, Weimer K, Muth ER, Enck P: Prediction of placebo responses: a systematic review of the literature. Front Psychol 2014; 5:1079. doi:10.3389/fpsyg.2014.01079

7. Jakšić N, Aukst-Margetić B, Jakovljević M: Does personality play a relevant role in the placebo effect? Psychiatr Danub 2013; 25:17-23

8. Lyby PS, Aslaksen PM, Flaten MA: Is fear of pain related to placebo analgesia? J Psychosom Res 2010; 68:369377. doi:10.1016/j.jpsychores.2009.10.009

9. Moerman DE, Jonas WB: Deconstructing the placebo effect and finding the meaning response. Ann Intern Med 2002; 136:471-476
10. Schienle A, Gremsl A, Übel S, Körner C: Testing the effects of a disgust placebo with eye tracking. Int $J$ Psychophysiol 2016a; 101:69-75. doi:10.1016/j.ijpsycho.2016.01.001

11. Schienle A, Übel S, Scharmüller W: Placebo treatment can alter primary visual cortex activity and connectivity. Neuroscience 2014; 263:125-129. doi:10.1016/j.neuroscience.2014.01.016

12. Schienle A, Übel S, Wabnegger A: When opposites lead to the same: a direct comparison of explicit and implicit disgust regulation via fMRI. Soc Cogn Affect Neurosci 2016b; 12:445-451. doi:10.1093/scan/nsw144

13. Schienle A, Walter B, Stark R, Vaitl D: Ein Fragebogen zur Erfassung der Ekelempfindlichkeit (FEE). Z Klin Psychol Psychother 2002; 31:110-120. doi:10.1026/0084-5345.31.2.110

14. Schweinhardt P, Seminowicz DA, Jaeger E, Duncan GH, Bushnell MC: The Anatomy of the Mesolimbic Reward System: A Link between Personality and the Placebo Analgesic Response. J Neurosci 2009; 29:4882-4887. doi:10.1523/JNEUROSCI.5634-08.2009

15. Scott DJ, Stohler CS, Egnatuk CM, Wang H, Koeppe RA, Zubieta J-K: Individual Differences in Reward Responding Explain Placebo-Induced Expectations and Effects. Neuron 2007; 55:325-336. doi:10.1016/j.neuron.2007.06.028.

16. van Laarhoven AIM, Vogelaar ML, Wilder-Smith $O H$, et al.: Induction of nocebo and placebo effects on itch and pain by verbal suggestions. Pain 2011; 152:1486-1494. doi:10.1016/j.pain.2011.01.043

17. Weimer K, Colloca L, Enck P: Placebo effects in psychiatry: mediators and moderators. The lancet Psychiatry 2015; 2:246-257. doi:10.1016/S2215-0366(14)00092-3

Correspondence:

Saša Zorjan, $M D$

Department of Clinical Psychology, University of Graz

Universitätsplatz 2/III, A-8010 Graz, Austria

E-mail: sasa.zorjan@um.si 